\title{
Study on dislocation and microstructural properties of friction stir welded joint for 2024-T4 aluminum alloys
}

\author{
Zhang Geming ${ }^{1}$, Liu Peng ${ }^{1 *}$, Shi Qingyu ${ }^{2}, \mathrm{Xu}_{\mathrm{Shubo}^{1}, \mathrm{Li} \mathrm{Jianing}^{1}, \text { Feng Keyun }^{1} \text {, Jing Cainian }}{ }^{1}$ \\ ${ }^{1}$ School of Materials Science and Engineering, Shandong Jianzhu University, Jinan 250101, P. R. China \\ ${ }^{2}$ Key Laboratory for Advanced Materials Processing Technology, Ministry of Education, \\ Department of Mechanical Engineering, Tsinghua University, Beijing 100084, P. R. China
}

Received 25 May 2016, received in revised form 12 September 2016, accepted 20 September 2016

\begin{abstract}
Microstructural properties and dislocation distribution of friction stir welded joint for 2024-T4 aluminum alloy were analyzed. Dislocations were mainly distributed in the grains of WNZ, mostly existed in the form of dislocation tangles and with a large number of precipitated phases. In TMAZ, dislocations existed in the grain boundary or grain interior in the form of dislocation pile-up. The dislocation density was also higher. Some dislocations existed near the grain boundary in the form of dislocation pile-up in $\mathrm{HAZ}$ along with some typical $\mathrm{AlCu}_{3}$ precipitated phase. In WN and TMAZ, the number and types of dislocation were not reduced along with the grain refinement because of the dynamic recovery and dynamic recrystallization process. The dislocation distribution was inconsistent with the hardness distribution of welded joints. The dislocation formed could be roughly divided into three phases: dynamic response process, dynamic recrystallization process and continuous deformation and recrystallization process.
\end{abstract}

K e y w or d s: 2024 aluminum alloy, friction stir welding, dislocations, microstructure, hardness

\section{Introduction}

As an important structural material, 2024 aluminum alloy has been widely used in the field of aeronautics and astronautics because of its high strength and heat resistance, such as aircraft manufacturing, rocket skin, fuel tanks, etc. However, corrosion resistance and weldability of 2024 aluminum alloy are poor. The joint also has some defects of porosity, hot crack and the corrosion resistance of the welded joint was decreased obviously using the general fusion welding method. Therefore, its application in the field of aeronautics and astronautics is severely restricted $[1,2]$.

Friction stir welding (FSW) is a new type of solid state bonding technology [3], which has been widely used in the welding of aluminum alloys. Furthermore, some good results have been obtained in recent years. FSW welding process can effectively avoid the defects such as a gas hole, thermal crack and so on, and get the high-quality joint [4-6], which has been widely used in the connection of light metal materials. The stir zone of FSW joint mainly consists of weld nugget zone (WNZ), thermo-mechanically affected zone (TMAZ), and heat affected zone (HAZ). At the same time, some researchers also divided the shoulder affected zone (SAZ) and the swirl defects zone (SDZ) $[7,8]$. At present, the study of friction stir welding of aluminum alloys indicates that the WNZ has a strong grain refining process [9-12], and the grain refinement is closely related to the dislocations distribution and its density in the process of hot plastic deformation [13]. The changes of the dislocation structure also directly affect the whole properties of the joints, besides it is also an important factor to promote the evolution of the metal structure. WNZ, TMAZ, SAZ, and HAZ in the stir zone are also undergoing different thermomechanical effects during the FSW. Therefore, it is necessary to observe and analyze the dislocation transition in these regions. This not only brings a more comprehensive understanding of the distribution char-

*Corresponding author: e-mail address: liupeng1286@163.com 
Ta b le 1. Chemical composition of 2024 aluminum alloy (wt.\%)

\begin{tabular}{ccccccc}
\hline Material & $\mathrm{Cu}$ & $\mathrm{Mg}$ & $\mathrm{Si}$ & $\mathrm{Fe}$ & $\mathrm{Mn}$ & $\mathrm{Al}$ \\
\hline $\mathrm{Al} 2024$ & $3.8-4.9$ & $1.2-1.8$ & 0.5 & 0.5 & $0.3-0.9$ & Rest \\
\hline
\end{tabular}

acteristics of the stir zone but also can analyze the reasons for the change of the microstructure and properties of the stir zone according to the angle of dislocation.

\section{Experimental}

The experimental material is 2024-T4 aluminum alloy sheet, and the main chemical composition of the test material is shown in Table 1 . The sample size is $150 \mathrm{~mm} \times 60 \mathrm{~mm} \times 3 \mathrm{~mm}$, and the butt friction stir welding was used. The experiment was carried out with a circular cylinder with a screw joint and a shoulder diameter of $18 \mathrm{~mm}$; the experimental sheet was fixed on the special steel fixture. During the experiment, the stirring pin was inserted into the joint part of the butt joint with the rotation of the $475 \mathrm{rev} \mathrm{min}^{-1}$, then respectively through different welding speed $\left(V=150-375 \mathrm{~mm} \mathrm{~min}^{-1}\right)$ along the welding direction. During the welding, the tilt angle between the stirring joint and the plane of the experimental plate was $2.5^{\circ}$, and the shoulder was pressed down to maintain $0.5 \mathrm{~mm}$. The pressing force of the holding mechanism was in a constant state during the welding process.

After welding, the samples were prepared into metallographic sample, and treated with low concentration mixed acid $1.0 \% \mathrm{HF}+1.5 \% \mathrm{HCl}+2.5 \% \mathrm{HNO}_{3}$ $+95 \% \mathrm{H}_{2} \mathrm{O}$. Then the microstructure of the joint was observed by optical microscope and transmission electron microscope (TEM) H-800. The microhardness distribution of the joint was conducted by microhardness experiment.

\section{Results and analysis}

\subsection{Microstructure}

The stir zone of 2024 aluminum alloy was observed by optical microscope, and the results are shown in Fig. 1. It can be seen that there is the obvious boundary between weld nugget (WN) and thermomechanical affected zone (TMAZ) (see Fig. 2a). The WNZ undergoes a high-temperature thermal cycle and ensures a strong stirring. The dynamic recrystallization occurs, and the microstructure of the grains and
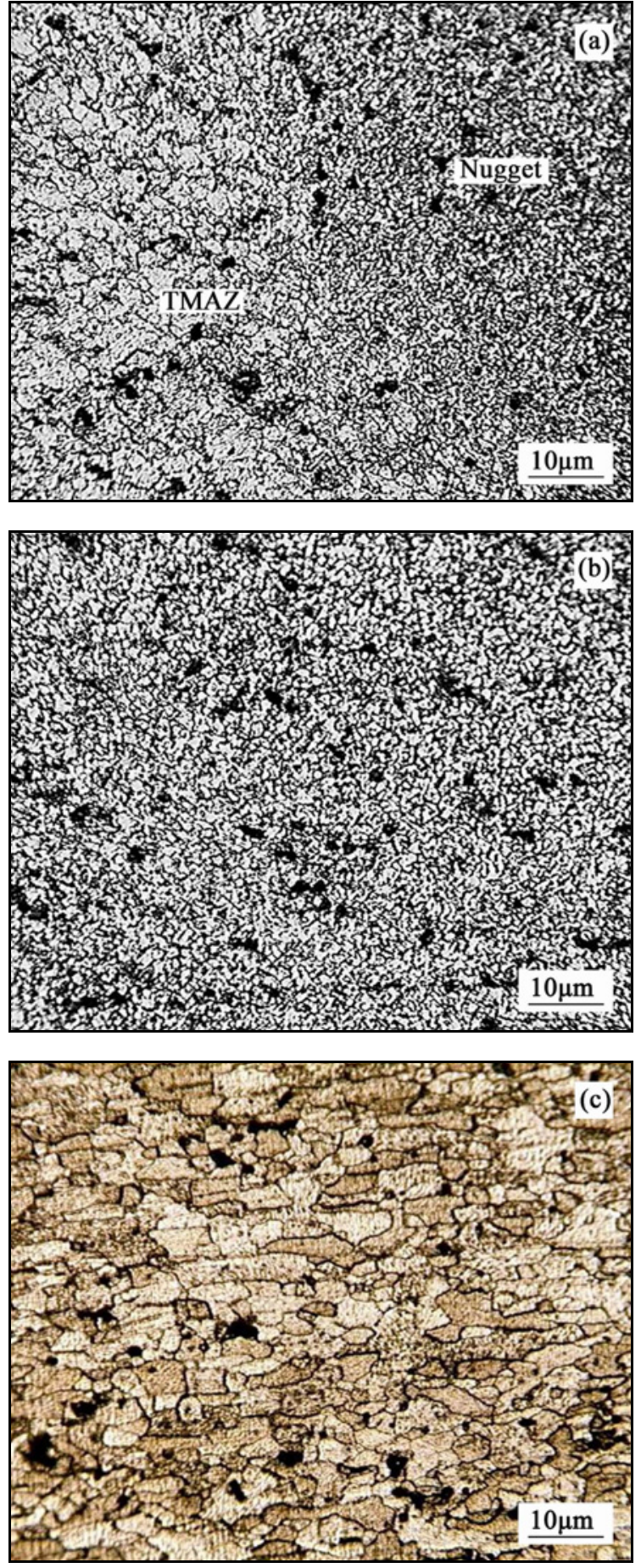

Fig. 1. Microstructure in the stir zone of FSW joint: (a) weld nugget zone/TMAZ, (b) weld nugget zone, and (c) heat affected zone.

the subgrains changed from the original panel to the equal axial grain (Fig. 2b). During the friction stir welding, the rotation of the stirring pin generates heat, which makes the base material to reach the plastic state, and the plastic flow of the material is under the mechanical action of the stirring pin. The plas- 

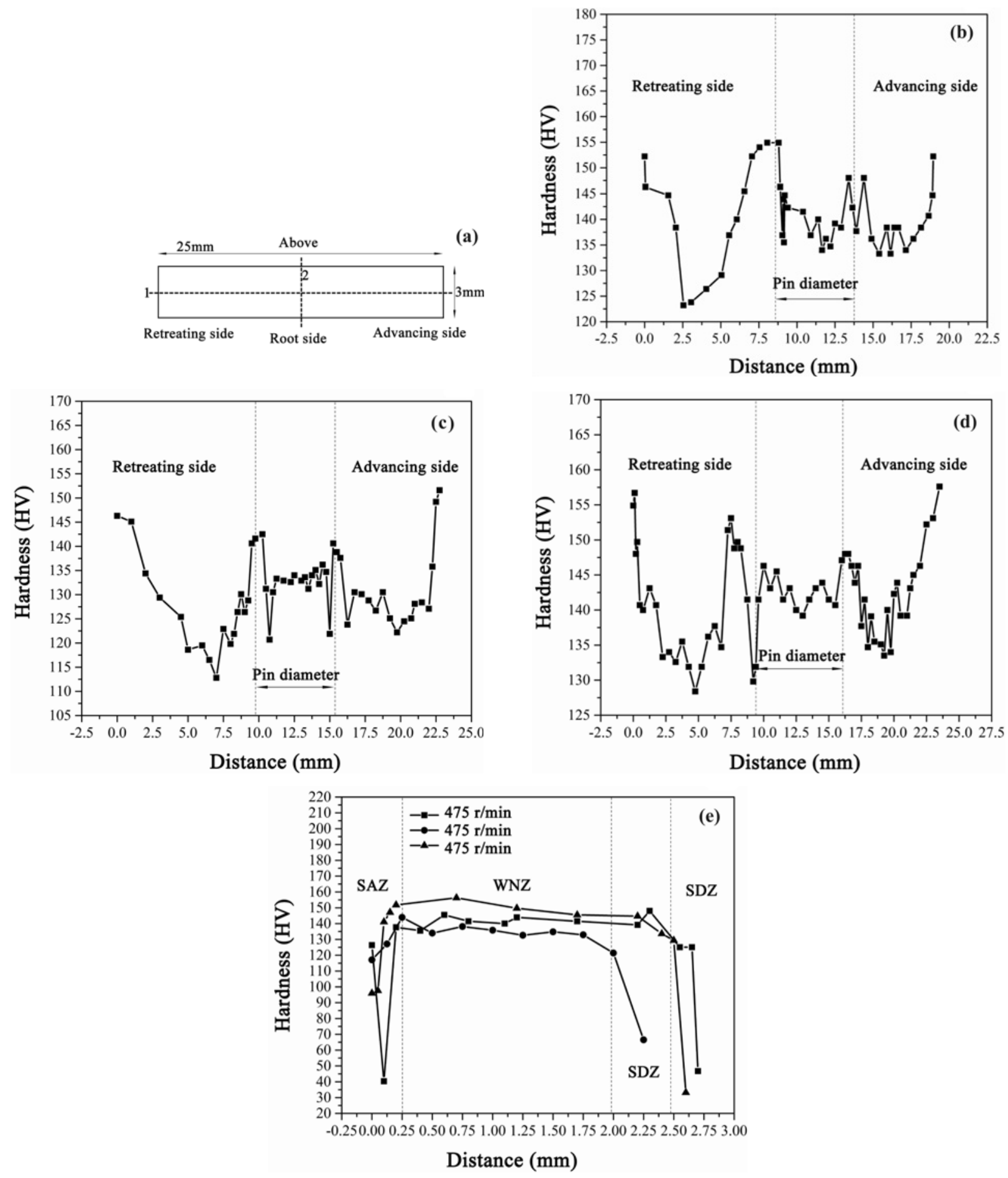

Fig. 2. The microhardness distribution of FSW joint of 2024 aluminum alloy: (a) schematic diagram of microhardness tested

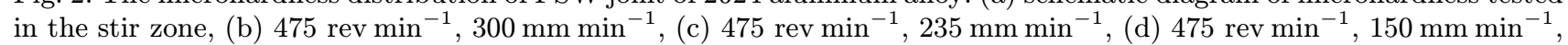
and (e) microhardness distribution in position 2 of Fig. 2a.

tic flow shows significant dynamic recrystallization. The welding zone is the area of the mechanical action of the stirring needle. At this time, the crystal grain plays the role of stirring needle and then is broken as it is too late to grow up, the formation of a relatively small axial grain structure occurs.
This phenomenon helps to improve the strength of the joint.

The TMAZ undergoes mechanical stirring, at the same time with significant thermal cycling effects. Shear force formed by mechanical stirring in the weld zone is easy to make the engine within the area of 

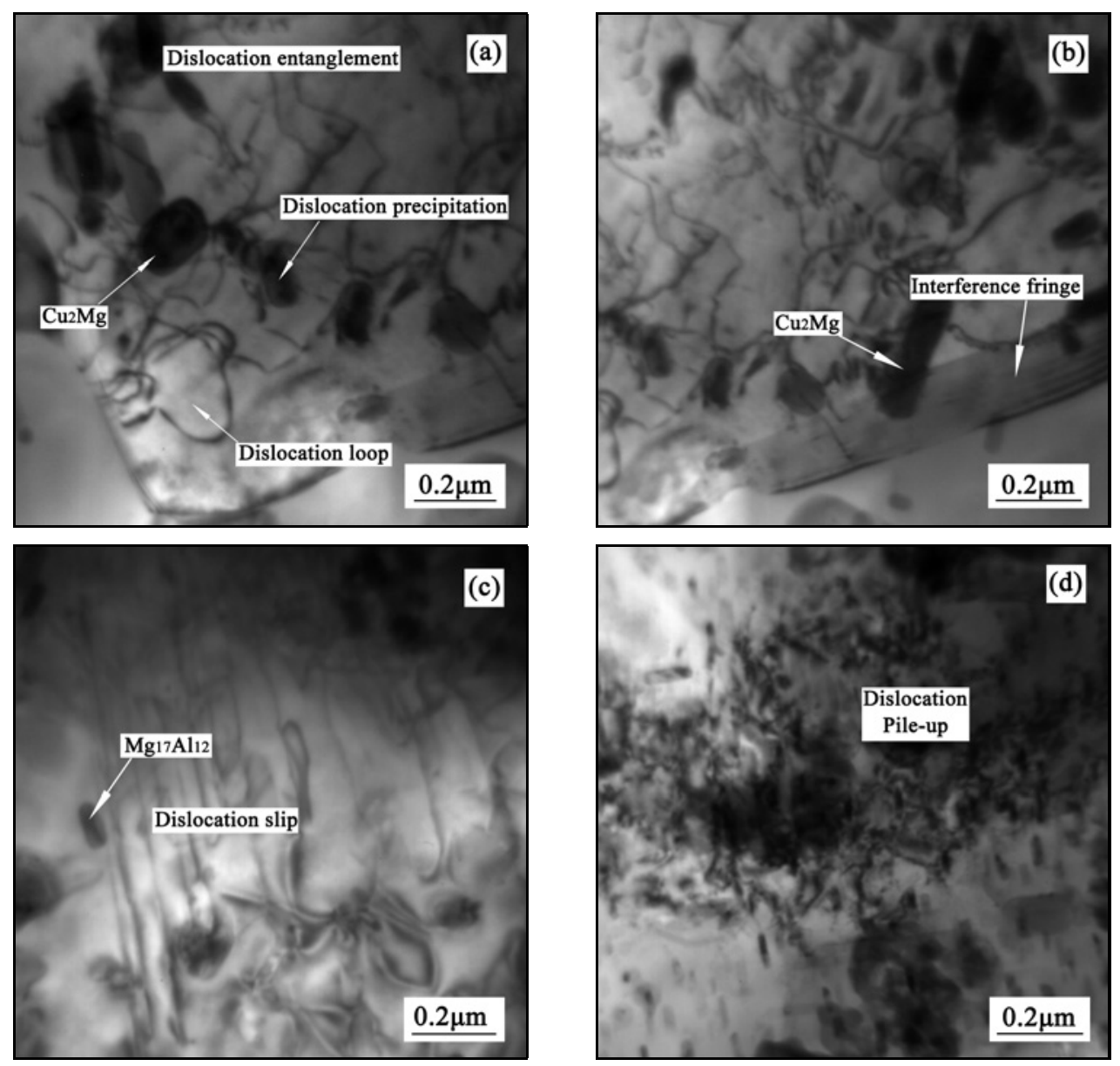

Fig. 3. TEM morphology of dislocations in WNZ and TMAZ for FSW joint: (a) dislocation entanglement, (b) precipitation phases, (c) dislocation slip, and (d) dislocation pile-up.

plastic deformation of the metal. Under the influence of the thermal cycle, the grains grow along the deformation direction. The grain size in this region is not uniform, and some grains have been deformed. But compared to the grain in HAZ (Fig. 2c), the grains of the TMAZ get a certain refinement.

\subsection{Microhardness distribution}

The microhardness distribution of FSW joints can directly reflect the changes in the various areas of the joint. MH-3 type microhardness experimenter was used in the experiment, load $25 \mathrm{~g}$, time $10 \mathrm{~s}$, along with the weld section of the specimen from the retreating side forward advancing side. The hardness experiment of the swirl defects zone (SDZ) from the lower part of the weld to the shoulder affected zone (SAZ) through the WNZ was preformed. The test position and results are shown in Fig. 2.

The experimental results indicate that the hardness distribution is basically similar under different conditions. The hardness distribution of RS to AS at the cross section is symmetrical, the microhardness of WNZ is slightly lower than that of TMAZ on both sides, but it is higher than that of HAZ. The hardness of HAZ back side is significantly higher than that of the forward side. The microhardness distribution along the thickness direction shows that the hardness of SDZ and SAZ is significantly lower than that of WNZ.

\subsection{Dislocations distribution in WNZ and TMAZ}

The microstructure and morphology of the typical dislocation structure by TEM analysis in WNZ and TMAZ of 2024 aluminum alloy FSW joints are shown in Fig. 3. It is observed that the dislocations in WNZ are mainly distributed in the grain interior, and most of them are in the form of dislocation entanglement. There is a large amount of precipitation and enhanced $\mathrm{Cu}_{2} \mathrm{Mg}$ [14], as shown in Fig. 3a. The stress interference structure that is easy to cause stress concentration near the grain boundary exists as well, as shown in Fig. 3b. In addition, in the strengthening precipitates and dislocation interaction region, the phenomenon of the dislocation motion is cut off or bypass enhanced precipitation phase can also be observed. 

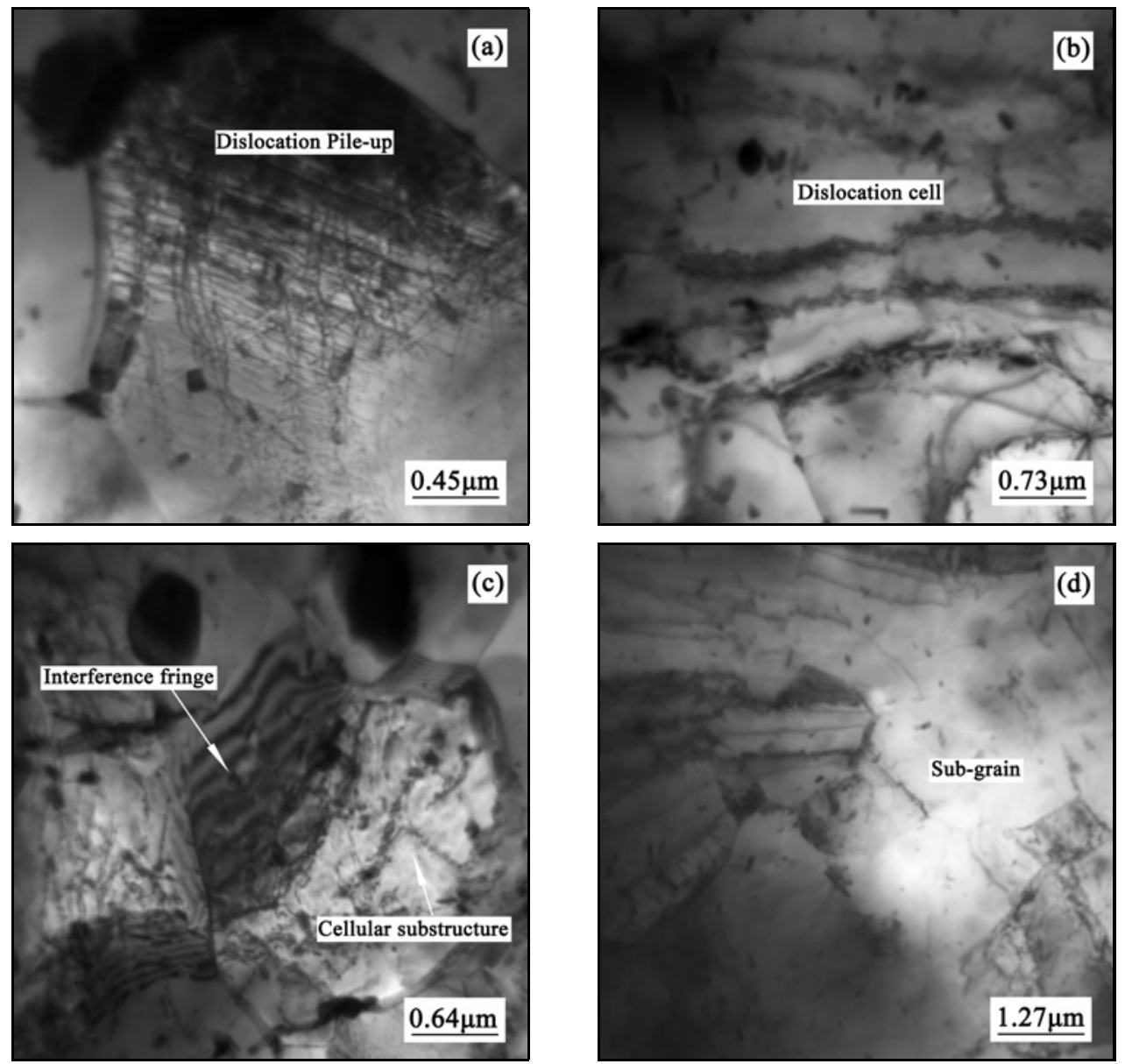

Fig. 4. Dislocation characteristic in SAZ of FSW joint of 2024 aluminum alloy: (a) grain internal dislocation, (b) dislocation cell, (c) stress interference, and (d) sub-grain structure.

The dislocation loop and the phase change caused by the cut can also be observed (see Fig. 3a). The typical slip lines formed after the dislocation slip are shown in Fig. 3c and the process of dislocation slip is accompanied with $\mathrm{Mg}_{17} \mathrm{Al}_{12}$ phase. Moreover, in the TMAZ, the dislocations distribution is not uniform, too. However, a large number of dislocations in the form of dislocation pile-up exist in grain boundaries or grains. The typical dislocation pile-up is shown in Fig. 3d.

According to the classical precipitation phase and dislocation strengthening mechanism, the movement of dislocations is in the grain during the larger plastic deformation. When the precipitation phase is enhanced, the dislocation bypasses the precipitated phase. At this time, both ends of the curved dislocation are attracted to each other, and the merge disappears. In the process of dislocation movement, the dislocation cuts off the precipitate when the precipitation is small, and then increases the phase interface area which leads to an increase in the interface energy. Therefore, the interaction of WNZ and precipitated phases is favorable for the enhancement of the strength of the region. However, the dislocation den- sity is low, and its distribution is not uniform, it will affect the strength of the region to a certain extent.

Figure 4 shows the microstructure of SAZ dislocation structure under TEM. The dislocation distribution in SAZ is very complicated, and the dislocation density is higher in the grain interior and the grain boundaries. The internal dislocation structure of the typical grain of SAZ is shown in Fig. 4a. A large number of mesh dislocation structures formed by the interaction of dislocations with different phases can be observed. However, the dislocation did not slip into the adjacent grains. It is shown in Fig. $4 \mathrm{~b}$ that the dislocation cell structure formed by dislocation slip in SAZ large scale grains. The dislocation density is higher in the vicinity of the cell wall, but the density of the dislocation is very low. The cellular substructure of the formation of dislocation cells in smallscale grains of SAZ is shown in Fig. 4c. Stress interference structure in the vicinity of the substructure grains is easy to cause stress concentration. The typical subgrain structure (less than $4 \mu \mathrm{m}$ ) was observed in SAZ in Fig. 4d and the angle of these subgrains was small. 

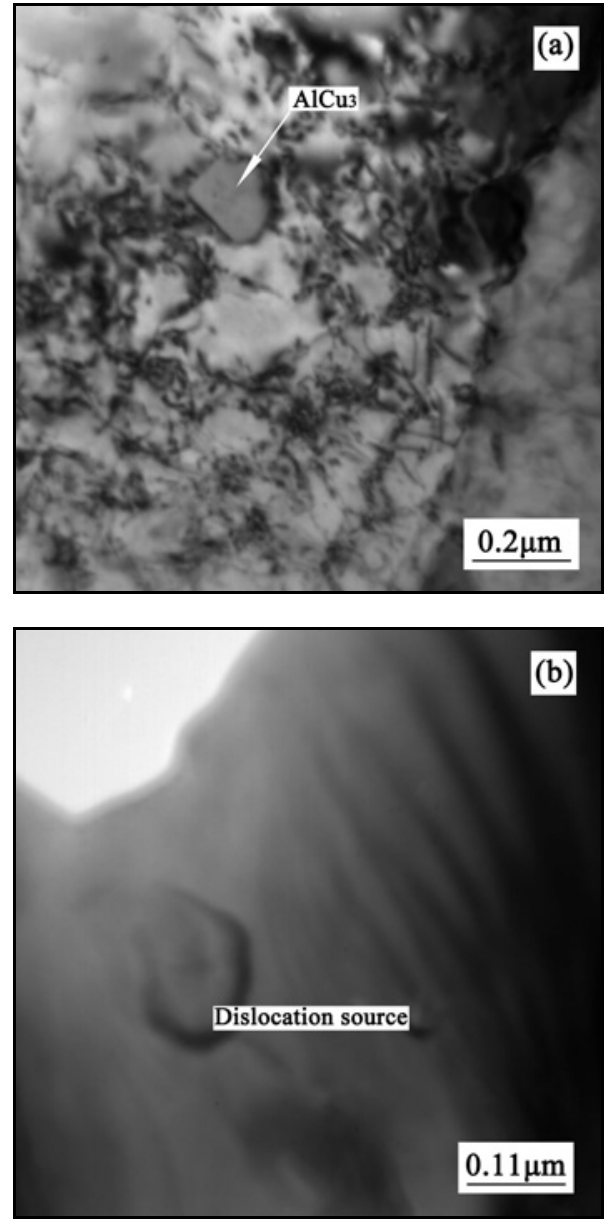

Fig. 5. Dislocation structure in HAZ of FSW joint of 2024 aluminum alloy by TEM: (a) dislocation in the grain boundary and (b) dislocation source.

\subsection{Dislocation distribution in the base metal and $\mathrm{HAZ}$}

The HAZ of FSW joint did not experience a large plastic stress, strain and high temperature, high pressure and other processes. However, the area is subjected to a certain welding thermal cycle; it leads to some changes of dislocations in this region. TEM experiment results indicate that the dislocation exists near the grain boundaries in the form of dislocation pile-up, as shown in Fig. 5a. The dislocation pile-up can also be observed in some $\mathrm{AlCu}_{3}$ precipitates [14]. Typical dislocation source in HAZ which leads to the dislocation motion is shown in Fig. 5b.

In the base metal, there are a large number of high-density dislocations in the grain boundary. At the same time, it is accompanied by a precipitation phase (see Fig. 6a). This is similar to the HAZ. However, the dislocation density in the base material is clearly higher than that of HAZ. The presence of a certain dislocation cellular substructure can be observed in the base material, the contour of the cell wall is not very clear (Fig. 6b). In addition, complex dislocation entanglement is formed in the crystal due to a large number of dislocations interactions. However, the precipitated phases are not observed in the presence of dislocation entanglement (Fig. 6c). The grain boundary precipitates and the typical grain boundary stress interference fringes around the grain boundaries are shown in Fig. 6d.

\subsection{Discussion}

\subsubsection{Effect of grain size}

Microstructure analysis of 2024 aluminum alloy FSW joint shows that the stirring zone (WNZ, TMAZ, and SAZ) experienced hot plastic deformation (the metal deformation temperature is higher than 0.5 $T_{\mathrm{m}}$, where $T_{\mathrm{m}}$ is the melting temperature), the metal grains have been significantly refined. There is a certain relationship between the metal material yield limit $\sigma_{\mathrm{s}}$ and the average grain size $d$ according to the classical theory of the relation between the metal strength and its grain size, so-called Hall-Petch [15], see the Eq. (1):

$$
\sigma_{\mathrm{s}}=\sigma_{0}+K d^{-\frac{1}{2}}
$$

Both $\sigma_{0}$ and $K$ in the Eq. (1) are determined by the constant of the material itself.

According to the Eq. (1), with the metal grain refinement, the strength of the metal and the plastic deformation will be improved. But according to the general theory of plastic deformation, the smaller the grain size, the dislocation pile-up group in metal will be less. As a result, a smaller stress concentration occurs in the vicinity of the grain boundary, which is very similar to the TEM observation in WNZ dislocation (see Fig. 3).

In the process of FSW, the metal in the hot plastic deformation zone (WNZ, TMAZ, and SAZ) is less recovered at the beginning of the plastic deformation. The size of the cellular structure, which is formed by dislocation movement and proliferation, is small. The dislocation entanglements in the cell wall (see Fig. 4) are caused by dislocation glide or climb in the process of metal deformation [16]. It is easy to lead to the formation of a large, poorly oriented cellular substructure due to the dislocation entanglement and accumulation with the increase of the strain rate provided by FSW. Moreover, the recrystallization processes through the cellular structure of the nucleus. At this time, the metal in stirring zone undergoes a continuous plastic deformation process, which makes the newly formed dislocation cell structure to further nucleation and growth. Eventually, it leads the metal of stirring zone to get more remarkably refined grain. 

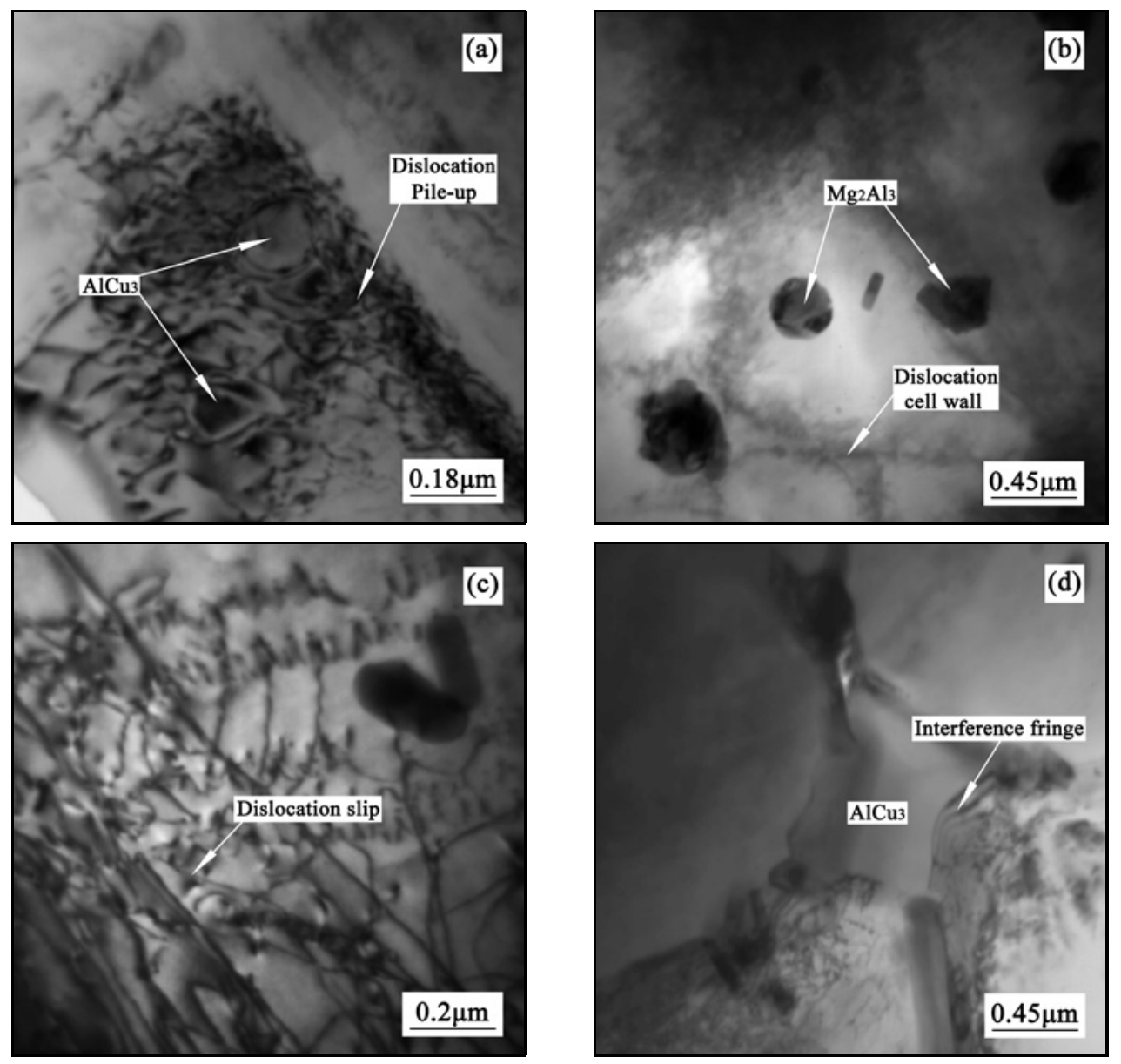

Fig. 6. TEM morphology of the dislocation structure of 2024 aluminum alloy: (a) precipitated phase, (b) dislocation cell wall, (c) dislocation pile-up, and (d) interference fringe.

The dynamic recrystallization process can be used to explain the refinement process of WNZ and SAZ. For TMAZ, the metallographic observation shows that the region has a significant deformation and the grain is elongated (see Fig. 1); there is no significant sign of being refined. However, TEM observations show that there are some grain refinement characteristics in the local area of TMAZ [14]; this produces a certain contradiction. Therefore, the plastic deformation process of the region may be more complex than that of $\mathrm{WNZ}$ and SAZ. According to the classical thermoplastic deformation theory, the dynamic recovery and dynamic recrystallization of the metal in the FSW process may be carried out simultaneously. Moreover, dynamic recrystallization may be carried out only in some regions near WNZ. When TMAZ metal occurs dynamic recovery, the metal has also experienced a pile-up of dislocations and dislocation tangles or the formation of the subcellular structure at the beginning of the deformation. When in the stable stage of deformation, the shape of the cellular structure may reach the equilibrium state. Therefore, it does not cause the nucleation and growth process but leads to the increase of the deformation degree. The grain shape is deformed in the main deformation direction, and the structure of the sub-crystal will be maintained.

TMAZ region can be observed with local grain refinement characteristics, indicating that there is a relationship between the FSW joint and the stir zone. However, due to the relatively narrow region, it is still not fully understood that the most elaborate microstructure is in the direction of length or thickness. At present, there are few studies in this area, and there is no more mature theory to explain the mechanism of tissue formation in this region. This is also one of the important research contents of this topic in the future.

\subsubsection{Effect of dislocations distribution}

WNZ is the most significant region of grain refinement, which is mainly dominated by the low-density dislocation entanglement in the grain (see Fig. 3a). The dislocation pile-up does not exist in the vicinity of grain boundaries. Based on the thermal theory of plastic deformation, dynamic recrystallization of WNZ can lead to metal softening and reduce its hardness. According to the microhardness analysis, 
the hardness of WNZ is lower than that of base metal (hardness difference is less than HV20). The hardness is higher than that on both sides of HAZ, and its distribution is relatively stable (see Fig. 2). In WNZ, a larger decrease in the hardness did not occur; it indicates that the strengthening mechanism of the interaction between the dislocation movement and the precipitation phase and the stress interference near the grain boundary (see Fig. 3b) can effectively improve the strength. After all, the region has a large number of enhanced precipitation phases, and high-density dislocations disappeared or reduced, the hardness of the region is lower than the hardness of the base metal.

TMAZ also experienced a higher hot plastic deformation, and the dynamic recovery process mainly occurs. Therefore, the region near the grain or grain boundary still retains a certain high dislocation density (see Fig. 3d). Besides, the observation of phase structure shows that the precipitation phase was formed with a certain strengthening effect $(\mathrm{AlCuMg}$ and $\mathrm{Mg}_{2} \mathrm{Si}$ ) [14]. These are the factors that cause the hardness of the region to be close to that of the base metal. For SAZ, the microstructure analysis shows that there was a significant grain refinement in the region. Although in the area of grain boundaries exists a large number of plastic deformations and the cellular structure caused by the dislocation movement (see Fig. 4d), this region still produces significant softening phenomenon and has not formed the precipitation strengthening effect due to the recrystallization process. This causes a significant reduction in the hardness of the region. The analysis of dislocations shows that the thermal plastic deformation process of SAZ can be more complex in the process of FSW. It is possible that the region is the intermediate state of the WNZ and TMAZ in thermoplastic deformation of FSW. However, at present, this phenomenon is only based on the analysis of dislocations, phase structure, etc., it still needs to be verified by thermodynamic and kinetic studies.

For HAZ, the dislocation structure has no significant changes produced only through the thermal cycle during the FSW. After welding, some typical dislocation structures which are similar to the base metal are still retained in this region. While the lower microhardness shows that the thermal welding cycle also promotes the movement of some dislocations, which leads to the decrease of high-density dislocations. Moreover, this is not the main reason for the decrease in the hardness of the region. According to the relevant analysis of phase structure in this region, a large number of the original enhanced precipitation phases of base material were gone through the phase transformation of the welding thermal cycle (the welding temperature of the area is higher than $\left.250^{\circ} \mathrm{C}\right)$. At the same time, there is no new phase which can play a role in strengthening. As a result, the strength of the region is reduced, which is the main fracture zone of the joint. According to the study of microstructure, dislocations and performance, combined with the classical theory of metal plastic deformation, the microstructure evolution of the metal in the FSW process of aluminum alloy can be obtained. The process microstructure evolution of 2024 aluminum alloy friction stir welding can be summarized in three stages:

Stage I: For aluminum alloy FSW, the rotating and advancing processes of the stirring joint make surrounding metal undergoing large plastic stress, strain, and temperature, hydrostatic pressure exerted on the shaft shoulder, etc. It leads to the proliferation of internal dislocation density of metal in the initial hardening process, furthermore, resulting in a partial dislocation of the entanglement and the formation of a cellular substructure. This process is a dynamic recovery process; the typical region is TMAZ.

Stage II: With the increase in the amount of metal deformation, dynamic recrystallization process happens in the stir zone, to promote the nucleation and growth in the dislocations cellular substructure. It also causes the partial dislocation to be absorbed by the grain, which leads to the generation of the grains. This process is also accompanied by some enhanced precipitation phase dissolution, phase transformation or precipitation. This is a dynamic recrystallization process. The typical regions are WNZ and SAZ.

Stage III: With the continuous process of friction stir welding, the continuous or discontinuous recrystallization process happens in the stir zone. The grains are fully refined, and this is a continuous deformation and recrystallization process. The typical region is WNZ. In the region of weak intense plastic deformation, characteristics of grain deformation retain in the dynamic recovery process, and the typical region is TMAZ

These three stages describe mainly the change of the metal structure during the hot plastic deformation of the stir zone. Compared with the TMAZ, in the area which is in direct contact with the stirring tool (SAZ and WNZ), grain structure, phase structure, and dislocation structure, still exist some differences. It shows that the shear strain caused by the stirring tool also plays an important role in the welding process in addition to the plastic deformation process.

\section{Conclusions}

This paper analyzed the microstructure, hardness, and dislocations distribution of friction stir welded joint of 2024 aluminum alloy. We try to go through the analysis of distribution characteristics of microdomain dislocations and discuss the formation of dislocations and its effect on microstructure and properties of FSW joint. 
(1) Some dislocations in WNZ are mainly distributed in the grain. Most of them are in the form of dislocation entanglement and accompanied by a large amount of precipitation enhanced precipitation phase $\mathrm{Cu}_{2} \mathrm{Mg}$. In the TMAZ, the dislocations distribution is not uniform, but a large number of dislocations exist at the grain boundaries meanwhile inside the grains it is in the form of dislocation pile-up. The dislocations distribution in SAZ is very complicated, and the dislocation density is higher in the grain and the grain boundaries. A large number of dislocation structures formed by the interaction of dislocations with different phases, the dislocations are not slipped into the adjacent grains.

(2) HAZ metal has not experienced large plastic stress, strain and high temperature, high pressure or other processes. The region is subject to a certain heat cycle. Dislocations in this region exist near the grain boundaries in the form of dislocation entanglement, along with some typical $\mathrm{AlCu}_{3}$ precipitates. However, the dislocation density of the base material was significantly higher than that in HAZ. In the base metal, there are some dislocation cellular structures formed during the aging treatment, and the cell wall profiles are not very clear.

(3) Dislocation analysis also indicated that the number and type of dislocations were not reduced with the decrease of grain size in WNZ and TMAZ. On the contrary, there are still many dislocations in two regions, which are related to the special dynamic recovery and dynamic recrystallization process of FSW. However, the distribution characteristics of dislocation and the hardness distribution of joint microdomain are basically consistent. The formation of dislocation during FSW process is roughly divided into three stages: dynamic recovery process, dynamic recrystallization process, and continuous deformation recrystallization process.

\section{Acknowledgements}

This research was financially supported by the Shandong Provincial Natural Science Foundation, China (Grant No. ZR2016JL017) and National Natural Science Foundation of China (Grant No. 51305240).

\section{References}

[1] Jeff, D.: Weld. J., 85, 2006, p. 42.

[2] Mironov, S., Inagaki, K., Sato, Y. S., Kokawa, H.: Metall. Mater. Trans. A, 46, 2015, p. 783. doi:10.1007/s11661-014-2651-0

[3] Thomas, W. M., Nicholas, E. D., Needham, J. C., Murch, M. G., Temple-Smith, P., Dawes, C. J.: International Patent Application No. PCT/GB92/02203 and GB Patent Application No. 9125978. 1991.

[4] Mahoney, M. W., Rhodes, C. G., Flintoff, J. G., Spurling, R. A., Bingle, W. H.: Metall. Mater. Trans. A, 29, 1998, p. 1955. doi:10.1007/s11661-998-0021-5

[5] Sato, Y. S., Kokawa, H., Enomoto, M.: Metall. Mater. Trans. A, 30, 1999, p. 2429. doi:10.1007/s11661-999-0251-1

[6] Salem, H. G., Reynolds, A. P., Lyons, J. S.: Scripta Mater., 46, 2002, p. 337. doi:10.1016/S1359-6462(01)01246-5

[7] Fratini, L., Buffa, G., Palmeri, D., Hua, J., Shivpuri, R. J.: Eng. Mater. Tech., 128, 2006, p. 428. doi:10.1115/1.2204946

[8] Li, J. N., Liu, P., Zhang, Y. B., Gong, S. L., Pan, G. H.: Kovove Mater., 53, 2015, p. 167. doi:10.4149/km-2015-3-167

[9] Kwon, Y. J., Shigematsu, I., Saito, N.: Mater. Trans., 44, 2003, p. 1343. doi:10.2320/matertrans.44.1343

[10] Kwon, Y. J., Shigematsu, I., Saito, N.: Scripta Mater., 49, 2003, p. 785. doi:10.1016/S1359-6462(03)00407-X

[11] Ma, Z. Y., Mishra, R. S., Mahoney, M. W.: Acta Mater., 50, 2002, p. 4419. doi:10.1016/S1359-6454(02)00278-1

[12] Su, J. Q., Nelson, T. W., Sterling, C. J.: J. Mater. Res., 18, 2003, p. 1757. doi:10.1557/JMR.2003.0243

[13] Woo, W., Balogh, L., Ungar, T., Choo, H., Feng, Z.: Mater. Sci. Eng. A, 498, 2008, p. 308. doi:10.1016/j.msea.2008.08.007

[14] Peng Liu, Guoliang Liu, Yan Deng, Jianing Li, Cainian Jing, Keyun Feng: Kovove Mater., 54, 2016, p. 363. doi:10.4149/km 20165363

[15] Zuev, L. B., Zarikovskaya, N. V., Fedosova, M. A.: Technical Phys., 55, 2010, p. 1299. doi:10.1134/S1063784210090112

[16] Jorge, A. M., Peres, M. M., Kiminami, C. S., Bolfarini, C., Botta, W. J.: Metall. Mater. Trans. A, 40, 2009, p. 3322. doi:10.1007/s11661-009-0054-4 